\title{
Metallosis: A New Form of Autoimmune/Autoinflammatory Syndrome Induced by Adjuvants Syndrome (ASIA)?
}

\author{
$\underline{\text { Rita Vaz }}{ }^{1}$, Pedro Xavier ${ }^{2}$, Sergio Brito ${ }^{1}$, Jorge Dantas ${ }^{1}$, Sofia Duque ${ }^{3,4}$, Jose Guimarães Consciência² Luis Campos $^{1,3}$ \\ ${ }^{1}$ Department of Internal Medicine, Hospital São Francisco Xavier, Occidental Lisbon Hospital Centre, Portugal \\ ${ }^{2}$ Orthogeriatric Unit, Orthopedics and Traumatology Department, São Francisco Xavier Hospital, Occidental Lisbon Hospital Centre, Portugal \\ ${ }^{3}$ Orthogeriatric Unit, Internal Medicine Department, São Francisco Xavier Hospital, Occidental Lisbon Hospital Centre, Portugal \\ ${ }^{4}$ University Geriatric Unit, Preventive Medicine and Public Health Institute, Faculty of Medicine, University of Lisbon, Portugal
}

\section{Doi: 10.12890/2019_001034 - European Journal of Case Reports in Internal Medicine - ๑ EFIM 2019}

Received: 29/12/2018

Accepted: 28/01/2019

Published: $25 / 02 / 2019$

How to cite this article: Vaz R, Xavier P, Brito S, Dantas J, Duque S, Guimarães Consciência J, Campos L. Metallosis: a new form of autoimmune/ autoinflammatory syndrome induced by adjuvants syndrome (ASIA)? EJCRIM 2019;6: doi:10.12890/2019_001034.

Conflicts of Interests: The Authors declare that there are no competing interests.

This article is licensed under a Commons Attribution Non-Commercial 4.0 License

\section{ABSTRACT}

Autoimmune/autoinflammatory syndrome induced by adjuvants (ASIA) is a new entity in which exposure to an adjuvant triggers an aberrant autoimmune response. Metallosis is a rare condition characterized by the deposition and build-up of metal debris in the soft tissues of the body associated with metal-on-metal (MOM) prosthetic devices. It can present with local/systemic symptoms and signs due to a chronic inflammatory host response. The authors present the case of a 51-year-old woman with a 6-month history of systemic complaints due to intoxication with metal ions from hip metallosis. This case highlights the importance of follow-up and continuous monitoring of patients with a hip prosthesis. As this is a rare condition, a patient presenting with unspecific symptoms such as ASIA syndrome induced by metallosis requires a high level of clinical suspicion as the removal of the adjuvant can resolve the condition.

\section{LEARNING POINTS}

- Asia syndrome is a new entity in which exposure to an adjuvant triggers an aberrant autoimmune response.

- Metallosis is an uncommon condition with local and systemic presentation.

- A high level of suspicion and close monitoring is needed in patients with vague systemic complaints.

\section{KEYWORDS}

ASIA syndrome, metallosis

\section{BACKGROUND}

Autoimmune/autoinflammatory syndrome induced by adjuvants (ASIA) is a new entity, described in 2011, in which exposure to an adjuvant triggers an aberrant autoimmune response. Initially, ASIA was associated with different conditions such as siliconosis, Gulf war syndrome (GWS), macrophagic myofasciitis syndrome (MMF) and post-vaccination phenomena. The diagnosis is made when two major criteria or one major criterion and two minor criteria are present (Table 1).

There is increasing concern in the scientific community concerning autoimmunity reactions induced by metals such as metallosis, defined as the deposition of heavy metal debris (cobalt and chromium primarily) within periprosthetic soft tissue and bone. It is caused by the presence of an articular prosthesis or the fracture of the articular component of a metal-on-metal (MOM) articular prosthetic device. 
The estimated incidence of metallosis is about $5 \%$ [1]. The deposition of heavy metal debris in soft tissue and bone induces the release of cytokines and chronic local inflammation. The local effects have been described as pseudotumours or osteolysis. The systemic effects are caused by an immunological response to metal debris, including constitutional symptoms such as nausea, vomiting and malaise, cognitive impairment, haematological abnormalities and neuromuscular changes. Risk factor for the development of metallosis include female gender, bilateral implants, high doses of corticosteroids, renal insufficiency and a suppressed immune system.

\section{CASE DESCRIPTION}

A 51-year-old woman was admitted to an orthopaedic ward for hip replacement surgery due to recurrent dislocation of a MOM hip prosthesis. Her past medical history included: seronegative axial spondyloarthritis with no radiological evidence and negative HLA B27, followed in a rheumatology outpatient clinic; hip replacement surgery 8 and 7 years previously, with MOM prostheses, due to coxarthrosis; peripheral eosinophilia of unknown cause followed in an immuno-allergology outpatient clinic; iron deficiency anaemia followed in a haematology outpatient clinic; and hospital admission 3 years previously to investigate a pseudotumour located in the psoas, iliac, gluteus and left quadriceps muscles, with unknown aetiology.

The patient had been referred to an internal medicine outpatient clinic, 1 month before the admission, because of a 6-month history of asthenia, anorexia, weight loss, headaches, nausea, vomiting, and hand and foot paraesthesia. The patient presented in a wheelchair due to instability and pain in her hip. She referred impaired memory, fatigue, difficulty concentrating and apathy, but denied other symptoms such as fever, chest pain, dyspnoea, abdominal pain, diarrhoea or constipation, alopecia, photophobia, xerostomia or xerophthalmia, oral ulcers, arthralgia or myalgia. Physical examination showed global grey discolouration of the skin and foot hypoesthesia. Blood analysis revealed hypokalaemia (3.12 mmol/l) and peripheral eosinophilia (1.50×10\%/l, 23.5\%).

During the hip replacement surgery, muscular rupture of the posterior two-thirds of the gluteus medius was observed together with blackening of the periprosthetic tissue with metal debris throughout compatible with metallosis (Fig. 1). Surgical debridement was performed and the hip prosthesis was replaced with a new one. Histology of the bursae was compatible with the xanthogranulomatous inflammatory process with histiocytic granulomas. Blood tests confirmed high levels of chromium $(7.6 \mu \mathrm{g} / \mathrm{l})$ and cobalt $(1.5 \mu \mathrm{g} / \mathrm{l})$, confirming the diagnosis of metallosis with systemic manifestations.

After retrospective review of the clinical case and the literature, the authors consider that the pseudotumour diagnosed in 2013 was actually an inflammatory mass related to metallosis.

As metallosis frequently presents with systemic manifestations, additional diagnostic examinations were ordered (transthoracic echocardiogram, cranial tomography and abdominal ultrasound) which were all normal. Ophthalmological examination showed no ocular involvement. The patient was discharged and during the 6-month follow-up, the global grey skin discolouration, foot paraesthesia, cognitive impairment, hypokalaemia and eosinophilia all resolved. 


\section{DISCUSSION}

In accordance with proposed criteria for the diagnosis of ASIA syndrome, our patient presented with four major criteria: exposure to an external stimulus, chronic fatigue, and neurological manifestations such as paraesthesia and cognitive impairment with memory loss which improved with removal of the MOM prosthesis. The atypical evolution that followed removal of the metal debris and the MOM prosthesis confirmed the diagnosis of ASIA syndrome induced by metallosis.

To our knowledge this is the first description of systemic features of autoinflammation in connection with metallosis.

As the number of patients submitted to hip replacement surgery is increasing, closer monitoring, with regular imaging, is required in case of prosthesis displacement or prosthesis fracture. The presence of systemic generic symptoms/complaints should lead to a high level of clinical suspicion of metallosis. Evaluation of blood levels of metal ions is mandatory and can confirm the diagnosis.

The development of symptomatic pseudotumours in patients with an articular prosthesis is uncommon and the consequent rate of revision of hip protheses is low (1.7-5.6\%). However, the presence of a pseudotumour in our patient 3 years before our diagnosis highlights the need for a high level of clinical suspicion for this diagnosis.

This clinical report demonstrates that foreign body reactions can underlie the clinical presentations of a systemic disease that could be lifethreatening. The growing use of prostheses makes this entity increasingly important.

\section{REFERENCES}

1. Oliveira C, Candelária I, Oliveira P, Figueiredo A, Caseiro-Alves F. Metallosis: a diagnosis not only in patients with metal-on-metal prostheses. Eur J Radiol Open 2015;2:3-6.

2. Sink E. Postponing total hip arthoplasty: the role for capsular arthroplasty in developmental dysplasia of the hip. JBJS Orthopaedic Highlights: Hip Surgery 2012;2:e5.

3. Amanatullah D, Sucher M, Bonadurer G, Pereira G, Taunton M. Metal in total hip arthroplasty: wear particles, biology, and diagnosis. Orthopedics 2016;39:371-379.

4. Birkett N, El-Daly I, Ibraheim H, Mbubaegbu C. Metallosis following full thickness wear in total hip arthroplasty. J Surg Case Rep 2015;2015:rjv122.

5. Loyo E, Jara L, López P, Puig A. Autoimmunity in connection with a metal implant: a case of autoimmune/autoinflammatory syndrome induced by adjuvants. Auto Immun Highlights 2012;4:33-38. 